\section{EN BUSCA DE LA GESTIÓN PARTICIPATIVA: EL CASO DEL PROYECTO DEL ECOBARRIO DE LA UNIÓN, LILLE, FRANCIA ${ }^{1}$}

María de Lourdes Flores Lucero²

\section{Resumen}

El proyecto piloto del ecobarrio de la Unión, en la Comunidad Urbana de la Metrópoli de Lille, Francia, intenta inscribirse en un ambicioso proceso de gestión participativa que ha llamado nuestro interés y sobre el que hemos realizado nuestro análisis. Diversos dispositivos y espacios de diálogo se han creado para permitir la interacción de los diferentes actores, y a pesar de que la influencia en la toma de decisiones se manifiesta lentamente, este proyecto se perfila como un ejemplo exitoso en dicha materia. El presente documento tiene como objetivo principal mostrar algunos resultados de nuestra investigación sobre el proceso

\section{LOOKING FOR PARTICIPATIVE NEGOTIATION: THE CASE OF THE UNION ECO- NEIGHBORHOOD PROJECT, LILLE, FRANCE ${ }^{1}$}

María de Lourdes Flores Lucero²

\begin{abstract}
The Union eco-neighborhood pilot project, in the Urban Community of Lille, France, has set up an ambitious process of participative negotiation that has drawn our interest, becoming the basis of this analysis. Various devices and spaces for dialogue have been created to allow interaction between different actors, and although the impact on decision making is slow, this project can be seen as a good example in the area of participative management. The main objective of this paper is to show some relevant results of the research on participative process to develop
\end{abstract}


participativo para elaborar y ejecutar el ecobarrio de la Unión, con el afán de abrir nuestra visión hacia otros contextos y experiencias donde también buscan desarrollar formas más democráticas en los proyectos urbanos. Utilizamos una metodología cualitativa y empírica basada principalmente en entrevistas a actores clave del proyecto, en observaciones de diversas dinámicas y en algunas participaciones en reuniones ciudadanas en torno al ecobarrio de la Unión, y a la revisión documental local e internacional en dicha materia.

\section{PALABRAS CLAVE: ECOBARRIO; GESTIÓN PARTICIPATIVA; LILLE; DESARROLLO SUSTENTABLE.}

Fecha de recepción: 09.03.12

Fecha de aceptación: 24.09.12

1 El presente trabajo es uno de los productos de la investigación posdoctoral (2010-2011) sobre la gestión de los ecobarrios en Francia, realizada en el Laboratorio de Territorio, Ciudades, Medioambiente y Sociedad de la Universidad de Ciencias y Tecnologías de Lille 1, con una beca del Programa Internacional Erasmus Mundus Lote 20.

2 México. Doctora en Gestión y Valoración Urbana por la Universidad Politécnica de Cataluña, Barcelona, España. Profesorainvestigadora del Doctorado en Procesos Territoriales y de la Maestría en Ordenamiento del Territorio de la Facultad de Arquitectura de la Benemérita Universidad Autónoma de Puebla, México. Email: lulu.lucero@gmail.com. and implement the Union eco-neighborhood, with the intention of opening our vision to other contexts and experiences which also seek to develop more democratic forms in urban projects. The research uses qualitative and empirical methods based on interviews with key stakeholders, observations of various dynamics (such as public meetings about the project) and local and international literature review in this domain.

\section{KEYWORDS: ECO-NEIGHBORHOOD; PARTICIPATIVE MANAGEMENT SUSTAINABLE} DEVELOPMENT.

Received: 09.03.12

Accepted: 24.09.12

1 This paper is the result of a postdoctoral research (2010-2011) on eco-neighborhood management in France carried out in the Territory, Cities, Environment and Society Research Unit at the Lille 1 University - Science and Technology funded by the Erasmus Program, lot 20.

2 Mexico. PhD in Urban Management and Valuation, Universidad Politécnica de Cataluña, Barcelona, Spain. Professor, Researcher, Doctorate Program on Territorial Processes and MA Program on Territorial Planning, Facultad de Arquitectura de la Benemérita Universidad Autónoma de Puebla, Mexico. Email: Iulu. lucero@gmail.com. project, Lille, France / María de Lourdes Flores Lucero 


\section{Introducción}

El desarrollo sustentable se manifiesta en las diferentes áreas del conocimiento como un cambio de ética, un concepto integrador y un principio de acción. Es decir, un nuevo reto histórico que genera nuevos debates científicos ${ }^{3}$ y donde enfrentar los problemas urbanos plantea nuevos cuestionamientos de cómo materializar los principios del desarrollo urbano sustentable a una escala abordable y en un marco democrático. En este contexto, los ecobarrios surgen como una respuesta a la búsqueda de la aplicación de los principios del desarrollo sustentable ya que permiten, por su escala, transformar la ciudad con la mirada y abordaje complejos de la sustentabilidad. Aunque la concepción más común sobre los ecobarrios se centra en aspectos tecnológicos-ecológicos, diversos autores ${ }^{4}$ coinciden en que éstos integran los elementos ambientales, sociales, económicos y de gobernabilidad participativa propuestos en las Agendas 21 locales; algunos ejemplos son las experiencias que nos muestra Hugh $^{5}$ en países como Alemania, Francia, Inglaterra, España, Estados Unidos, Canadá, etc. En la mayor parte de los ejemplos de ecobarrio, la parti-

\footnotetext{
3 Da Cunha, 2005.

4 Hugh, 2000; Souami, 2009; Charlot-Valdieu y Outrequin, 2009; Lefévre y Sabard, 2009, entre otros.

5 Hugh, 2000.
}

cipación social ha sido fundamental para fortalecer los procesos democráticos en la elaboración y ejecución de los proyectos, ya que, como bien lo indica Ascher", "la gobernabilidad urbana supone un enriquecimiento de la democracia representativa por nuevos procedimientos deliberativos y consultivos" transformadores de la democracia participativa. Esta última requiere, de acuerdo con Morin', inventarse a partir de las experiencias locales en las cuales los ciudadanos participen en los debates públicos y dar lugar, además de las reuniones ligadas a los proyectos públicos, a los consejos de barrio, los que podrían actuar como jurados de las acciones de orden público. Su virtud radica en el poder de volver concretas las decisiones sobre problemas concretos, revitalizar el espíritu de la comunidad, solidaridad y responsabilidad, y la regeneración del civismo donde se fermenten las buenas voluntades que han sido subempleadas. No obstante, Morin ${ }^{8}$ nos alerta sobre uno de los riesgos de la democracia participativa: la subrepresentación cuando es fuerte la abstinencia de participación ciudadana.

En algunos países como Francia, la construcción de ecobarrios ha sido creciente en los últimos

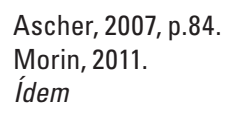


años ${ }^{9}$, sobre todo a partir del mandato de la Gre$n$ lle $^{10}$ del Medioambiente, donde se solicitaba la construcción de un ecobarrio antes del 2012 en todas las comunas que tuvieran programas de desarrollo del hábitat significativo y se reforzaba la política de la A2l en dicha materia.

Varios gobiernos locales atendieron a dicho mandato, entre ellos la Comunidad Urbana de la Metrópoli de Lille (LMCU por sus siglas en francés). Desde la Agenda 21 de Lille, los ecobarrios se han planteado como una prioridad y "la ocasión de demostrar a la escala de uno o más sitios pilotos, la viabilidad operacional de los principios de desarrollo sustentable"11. En este contexto y con la voluntad política de la LMCU, las autoridades de Lille propusieron en 2006 la construcción de un ecobarrio piloto en la ex zona industrial de la Unión.

El proyecto de ecobarrio de la Unión se ha planteado como un lugar de experimentación que además de responder a los principios medioambientales, sociales y económicos propios del desarrollo sustentable, representa una oportunidad para la construcción de espacio para desarrollar ejercicios de

9 De acuerdo a Souami (2009, p.203), hasta el 2008 había 57 proyectos de ecobarrios (barrios sustentables) en Francia y según el Gobierno de Francia (en línea, 2011a), en 2009 se registraron 160 proyectos en la convocatoria nacional de proyectos de ecobarrios.

10 Ley sobre el medioambiente en Francia, en línea (Gobierno de Francia, 2011b).

11 Lefévre y Sabard, 2009, p.150.

206 revista invi № 76 / Noviembre 2012 / Volumen N ${ }^{0} 27:$ 203-221 nuevas formas de gestión participativa y de aprendizaje colectivo. En este último rubro, los actores políticos de Lille han expresado su voluntad para construir el ecobarrio de manera conjunta con los actores involucrados. Sin embargo, la inexperiencia en esta materia fue uno de los primeros retos a enfrentar por parte de las autoridades y el inicio de la construcción conjunta de una nueva forma de hacer ciudad, incluyendo, por supuesto, conflictos entre los diferentes actores, llevándolos a ensayar diferentes caminos hacia la democracia participativa. Ante esta situación, nos hemos planteado observar y analizar los procesos participativos que se están desarrollando en el ecobarrio de La Unión.

Para ello nos basamos en una metodología cualitativa basada en la realización de entrevistas a algunos funcionarios públicos de la Comunidad Urbana de la Metrópoli de Lille, a representantes de la Sociedad de Economía Mixta (SEM, promotor del proyecto) y a miembros del Colectivo de la Unión. También observamos diversos procesos de consulta pública oficiales y asistimos a varios de los talleres mensuales informativos y educativos, organizados por la SEM 
en el Taller eléctrico, sobre el ecobarrio de la Unión. Participamos en reuniones del Colectivo de la Unión (observación participante) y asistimos a eventos informativos y de debate público organizados por este último, todo lo anterior en un marco de investigación-acción. Nuestro enfoque teórico para analizar dichos procesos se ha basado en los principios del desarrollo sustentable, enfatizando en la gestión participativa como elemento fundamental para la concertación y negociación entre los diferentes actores involucrados en el proyecto. Revisamos y analizamos los documentos locales oficiales en torno al proyecto (publicados la mayoría en las páginas web oficiales) y realizamos visitas de campo al sitio donde se está desarrollando el futuro ecobarrio. El análisis de los elementos teóricos y empíricos de nuestro objeto de estudio nos permitieron aproximarnos al entendimiento de los procesos participativos en torno al mencionado ecobarrio y establecer inferencias para llegar a las reflexiones finales planteadas.

\section{El ecobarrio de la Unión, un proyecto de reconversión urbana en el marco del desarrollo sustentable}

El proyecto de ecobarrio de la Unión se ubica sobre una zona intercomunal (que comparten las comunas de Tourcoing, Roubaix y Wattrelos), con un pasado industrial y obrero que data de mediados del siglo XIX cuando se instalaron industrias textiles y más tarde cerveceras y petroquímicas. En los años sesenta empezó el estado de abandono de dicha zona, dejando como huella la contaminación de suelos, desempleo y precariedad. Estas características de no durabilidad aunadas a su favorable posición geográfica transfronteriza hicieron de la Unión el lugar ideal para desarrollar un proyecto de ecobarrio experimental, de transformación y renovación urbanas donde se pudieran aplicar los principios del desarrollo sustentable.

El futuro ecobarrio de la Unión se inscribe en una política de desarrollo sustentable con un plazo de quince años a partir del 2006. Los componentes medioambiental, social y económico son abordados desde diferentes perspectivas en el proyecto. Así, encontramos por ejemplo que la descontaminación de terrenos, la limitación de la producción de desechos, la organización del ciclo del agua, la gestión de los desplazamientos, la priorización a la movilidad suave y los objetivos de Alta Calidad Medioambiental (HQE por sus siglas en francés) en los edificios, forman parte del objetivo medioambiental. El componente social prevé la mezcla y diversidad social, el 30\% de vivienda social, densificación urbana, y la accesibilidad a los equipamientos y servicios de proximidad. En el componente económico se plantea crear un polo de excelencia y dos filiales: La Plaine image y Los Textiles Técnicos, estos últimos organizados alrededor del Centro Europeo de Textiles Innovadores 
-CETI- (cuya inauguración se prevé para el presente año, 2012) y localizados en la "plataforma urbana" de actividades y hábitat (elemento motor del proyecto). En el ecobarrio se prevé dos grandes proyectos: un parque urbano de quince hectáreas y los estacionamientos silos ${ }^{12}$ de La Unión. Además, la Zona de Ordenamiento Concertado (ZAC por sus siglas en francés $)^{13}$ de La Unión está destinada para albergar alrededor de 3000 habitantes y 6000 trabajadores, y cuenta con un financiamiento de 173 millones de euros ${ }^{14}$.

El ecobarrio de la Unión es un proyecto ambicioso y complejo donde intervienen diversos actores entre los que destacan la Comunidad Urbana de la Metrópoli de Lille (LMCU por sus siglas en francés), que junto con el promotor del proyecto -integrado por la Sociedad de Economía Mixta

12 Los estacionamientos silos son compartidos entre los habitantes y las empresas. Cumplen diferentes funciones, como estacionamientos para vehículos, punto de estacionamiento, reparación de bicicletas, servicio de autos compartidos, almacenamiento de aguas pluviales, producción local de energía, etc. (Société d'Economie Mixte, 2011).

13 Las ZAC son herramientas para el ordenamiento del espacio urbano mediante las cuales se elaboran proyectos urbanos (desde la adquisición de terrenos hasta la realización de equipamientos públicos y construcciones) en concertación con la población local (Gobierno de Francia, 2011).

14 Université Populaire et Citoyenne, 2011, p.6.

208 revista invi № 76 / Noviembre 2012 / Volumen N N 27: 203-221
(SEM) - Ciudad Renovada y la Sociedad Anónima de Economía Mixta Euralille son los encargados de la gestión del proyecto. El contratista es la empresa Reichen E Robert \& Asociados. También interviene el Consejo Intercomunal formado por las tres comunas involucradas donde se realiza el proyecto y diversas asociaciones ${ }^{15}$ entre las que destaca el Colectivo de la Unión ${ }^{16}$ que juega un rol muy importante de interpelación entre los habitantes y los responsables de la gestión del proyecto. El objetivo conjunto de los actores en el ámbito de gobernabilidad es el mismo: construir un ecobarrio ejemplar en materia de desarrollo sustentable mediante procesos de gobernabilidad participativa ${ }^{17}$. Estos procesos, como lo indica Simon ${ }^{18}$, se construyen en común, ya que el desarrollo sustentable no pertenece a nadie, ni a los economistas, ni a los ecologistas, ni a los ingenieros, ni a los sociólogos, ni

15 Por ejemplo, la Asociación Ciudadana de Residentes del Oeste de Wattrelos (ACROW), Los ancianos de la Tossée, Los amigos del Canal, etc.

16 El espíritu participativo de los habitantes de Roubaix hizo que en 2005 los antiguos asalariados, habitantes de los barrios circunvecinos, desempleados y otros formaran el Colectivo de la Unión (CU), que cuenta con el apoyo de los miembros de la Universidad Popular y Ciudadana. Hasta el 2011, el CU contaba con aproximadamente 500 adherentes.

17 Existen 4 etapas en el proceso participativo: 1) información: solo se informa del proyecto a realizar; 2) consulta, se recoge la opinión de los ciudadanos para ayudar a esclarecer las decisiones de las autoridades; 3) concertación, permite el trabajo conjunto entre actores para la apropiación y aceptación del proyecto y 4) coproducción, se comparte el poder decisional (CERTU, 2006, p.9).

18 Simon, 2003. 
a los políticos. Es gracias a una gestión concertada que se construyen los proyectos de infraestructura del territorio con bases duraderas. Además, algunos autores que han realizado estudios en América Latina, como Iracheta ${ }^{19}$ y Ziccardi ${ }^{20}$, nos recuerdan que para la construcción de una gobernabilidad democrática es necesario que los gobiernos de las ciudades ofrezcan las condiciones para que existan los espacios e instrumentos para la participación ciudadana. Bajo esta mirada, el ecobarrio de La Unión presenta como una de sus características de reconocimiento la concertación creciente con los habitantes. Por su parte, la LMCU inscribe el proyecto en una dinámica transversal y en un proceso de concertación formalizados mediante el Referencial dinámico de desarrollo sustentable (2010) que se desprende de la Agenda 21 (2006) y de la Carta Ecobarrio (2010), ambas de la Metrópoli de Lille.

\section{El difícil inicio del proyecto: la experiencia del barrio Stéphenson}

Al inicio, el proyecto de la Unión comprendía el barrio Stéphenson (que incluye las calles Stéphenson y Tossée), donde se encuentran ubicadas pequeñas casas de los años 30. "Originalmente, el proyecto

\footnotetext{
19 Iracheta, 2008

20 Ziccardi, 1996.
}

de la Unión iba a arrasar con las viviendas existentes en el sitio, la idea original era construir un proyecto completamente nuevo"21. La decisión autoritaria que se tomó sin consultar a los habitantes generó un fuerte conflicto entre las autoridades y los habitantes. Sin previo aviso, una noche de mayo de 2000, los habitantes fueron informados de que debían irse. "Ese día se nos vino el mundo encima", cuentan los habitantes de Tossée. Rápidamente, los lugareños se reunieron y decidieron crear una asociación llamada "No arrases con mi barrio", "Rase pas mon quartier"22. El conflicto era claro, por un lado las autoridades querían demoler las casas y por el otro los habitantes no estaban dispuestos a partir.

No arrases con mi barrio estaba formado por aproximadamente sesenta familias directamente afectadas y otras solidarias que formaban el grupo de la resistencia; el resto decidió ser indemnizado y partir $^{23}$. Cuatro años de lucha intensa y mucha mediatización dieron lugar a una situación crítica que llevó al alcalde y a su equipo a redefinir los límites territoriales del proyecto y decidir que el barrio Stéphenson no se inscribiría dentro del ecobarrio y por lo tanto no se demolerían las casas, que además serían objeto de una rehabilitación. No se trató pues de una voluntad política para integrar las

\footnotetext{
21 Leromain, 2010, p. 49.

22 Société d'Economie Mixte, 2010, p.7.

23 Ídem p.8.
} 
opiniones de las personas sino de una respuesta a la presión ciudadana organizada. No obstante es un hecho que los políticos y empleados del sector público recuerdan como un acto de tomar en cuenta las peticiones ciudadanas y de una voluntad política para integrar la opinión de los habitantes en el desarrollo del proyecto, mientras que los habitantes de las calles Stéphenson y Tossée lo recuerdan como una lucha intensa que finalmente ganaron.

\section{Los nuevos dispositivos para la participación}

La experiencia de las calles Stéphenson y Tossée dejó una huella profunda entre las autoridades, técnicos y habitantes, quienes sabían que, como anota Tamayo ${ }^{24}$, "los proyectos de modernidad (futuros alternativos), al final, cualesquiera que sean, se derivan de una combinación dialéctica entre acción y reflexión colectivas. Para ello era necesario y urgente crear dispositivos y espacios que permitieran la participación y el diálogo entre los diferentes actores para interactuar, aprender y tomar decisiones en torno al proyecto".

24 Tamayo, 1998, p.163.

210 revista invi № 76 / Noviembre 2012 / Volumen N ${ }^{\circ} 27: 203-221$
Así, sin ninguna duda se creó el "Taller eléctrico" (Atelier électrique), ubicado en la calle Tossée, como espacio para la coproducción ${ }^{25}$ para la rehabilitación de las casas que iban a ser demolidas ${ }^{26}$. Actualmente es la única zona objeto de rehabilitación, en contraparte al proceso del barrio de la Unión ${ }^{27}$. Este taller también sirve como un espacio de información y diálogo donde cada mes se realizan conferencias públicas que abordan temas relacionados con el proyecto, por ejemplo la recolección de basura, jardinería, construcción del proyecto, el agua, etc.

Además del Taller eléctrico, la SEM, como encargada y mediadora del proyecto, ha retomado las estructuras existentes como los consejos y comités de barrio para llevar a cabo el proceso participativo. Además creó tres dispositivos para mejorar y estrechar la comunicación entre las autoridades y los habitantes: un dispositivo para la comunicación cuyo objetivo es el de comunicar al público en general sobre todas las acciones que se desarrollan en torno al proyecto; otro para la participación y desarrollo sustentable cuyo objetivo es asociar a los diferentes actores (habitantes y futuros habitantes, empresas, autoridades, etc.) durante todas las etapas del proyecto, este dispositivo es en el

25 La coproducción para el ecobarrio de la Unión no es un objetivo manifestado en ningún momento por los actores públicos.

26 Este trabajo puso en el centro del proyecto de rehabilitación de las casas a los habitantes, quienes trabajaron directamente con el arquitecto de renombre Bouchain.

27 Leromain, 2010, p.49. 
que se desarrollan más dinámicas participativas; y el dispositivo cultura, que tiene como objetivo dar los medios a través de los cuales los habitantes se apropien del proyecto (por ejemplo, a través de encuentros, de las reuniones en el taller eléctrico, etc). Estos dispositivos están fuera de los procesos habituales de los proyectos y cada uno requiere de nuevo personal con competencias y habilidades para la negociación y gestión del proyecto. Este hecho significa más requerimientos financieros e identificar a personas clave cuyas características de personalidad y conocimiento del entorno beneficien la mediación del proyecto.

\section{Las instancias de la gobernabilidad}

La organización para coordinar los trabajos entre las autoridades, los técnicos y usuarios (habitantes y asociaciones) de la Unión, gira en torno a cuatro instancias: 1) el Comité de Pilotaje, que orquesta la ejecución del proyecto y está formado por los tres alcaldes de las comunas involucradas, la Comunidad Urbana de la Metrópoli de Lille (LMCU por sus siglas en francés) y los desarrolladores del proyecto. 2) El Comité Consultivo, representado por la SEM, es el que provee la información sobre el avance del proyecto ante las asociaciones y los representantes de los habitantes. Este comité es el que juega un rol primordial en la sensibilización de los habitantes y futuros usuarios para participar en el proyecto. 3) Los grupos de trabajo constituidos por los usuarios (habitantes, futuros habitantes, asalariados, representantes de las asociaciones, etc.) y los técnicos de los diferentes servicios municipales. Los grupos de trabajo son el corazón de la participación social y desde donde surgen las propuestas para mejorar el proyecto. Estos grupos tienen como objetivo intervenir en la fase previa al proyecto y trabajar en interacción con el comité de pilotaje. 4) Los grupos del proyecto, formados por las mismas personas de los grupos de trabajo, tienen como objetivo la puesta en marcha de las acciones definidas por los grupos de trabajo y validadas por el comité de pilotaje. Finalmente, para reunir a las diferentes instancias, se han creado Las Asambleas de la Unión que les permiten una vez por año encontrarse para saber el avance y la evolución del proyecto. Estos innovadores espacios representan lo que Callon ha llamado foros híbridos ${ }^{28}$, ya que en ellos convergen personalidades distintas, como políticos, técnicos, representantes y habitantes interesados en las problemáticas abordadas. Estos espacios parecen representar una puerta hacia nuevas formas de construcción de la democracia.

28 Los foros híbridos son espacios abiertos donde grupos heterogéneos pueden movilizarse para debatir aspectos técnicos que interesan al colectivo (Callon, 2001, p. 36).

revista invi № 76 / Noviembre 2012 / Volumen $N^{0} 27$ : 203-221

211 
Entre las principales realizaciones, por el momento, de los grupos de trabajo están: el referencial dinámico del desarrollo, documento que presenta el conjunto de las ambiciones económicas, sociales y medioambientales del ecobarrio, las conversaciones públicas realizadas en el Taller eléctrico ${ }^{29}$, el estudio de contaminación ${ }^{30}$ realizado ante la inminente contaminación de suelos debido al pasado industrial de la zona y la vigía producto de la reflexión sobre la gestión evolutiva del proyecto, formada por una asociación rubesiana y algunos trabajadores del sector, quienes a través de su presencia cotidiana en el lugar pueden observar y señalar los aspectos disfuncionales, como la recogida de basura, intrusiones en los edificios cerrados, etc., para que el desarrollador pueda atender las problemáticas identificadas ${ }^{31}$

No obstante, dichas realizaciones se quedan cortas ante algunos hechos sobre el desarrollo del proyecto, por ejemplo el proceso participativo de algunos proyectos como el CETI o la ampliación de la calle de la Unión se limitaron a informar sobre las acciones que se iban a ejecutar y a escuchar las opiniones de los asistentes. Es decir, la participación se ha limitado a las etapas de información

29 Para escuchar algunas conversaciones, dirigirse a la página web http://www.lunion.org/eco-quartier/realisations/conversations-publiques.html

30 Se han realizado 510 estudios en toda la zona del proyecto (la Unión, 2011).

31 La Unión, 2011

212 revista invi № 76 / Noviembre 2012 / Volumen N ${ }^{0} 27:$ 203-221 y de consulta. Sin embargo, no hubo una amplia discusión conjunta entre los diferentes actores que les permitiera llegar a la concertación, a pesar de los dispositivos e instancias para la participación antes mencionados.

\section{Las limitantes de la participación}

La creación de los dispositivos para la participación abrió una nueva perspectiva en esta materia por parte de todos los actores, sin embargo hemos detectado algunas limitantes que han aletargado su plena puesta en práctica. Entre las más destacadas encontramos: a) la posición de arbitraje del comité de pilotaje, b) la multiplicación de espacios de diálogo y subutilización de los existentes, c) medios financieros limitados, d) las diferencias lingüísticas entre técnicos y ciudadanos, e) un marco reglamentario limitante $y f$ ) un proyecto intercomunal difícil de gestionar.

a) La posición de arbitraje del comité de pilotaje. Aunque el comité de pilotaje es portador de la última palabra, éste ha jugado un rol más bien de árbitro ya que dentro del comité no existe representación 
ciudadana cerrando así la principal puerta decisional del proyecto a los ciudadanos. Además, según el Colectivo de la Unión, el contacto con el grupo de pilotaje no siempre es fácil, por lo que hay que presionar o buscar otros medios para presentarles las propuestas ciudadanas.

Por su parte, el comité de pilotaje está obligado a defender su postura política pues sabe que el ecobarrio de la Unión es un proyecto político que debe ser compartido con los habitantes pero también asumido por los políticos. Esta postura lleva a las autoridades a tomar la última decisión contradiciendo los principios de la concertación ${ }^{32}$.

Por ejemplo, en marzo de 2009 la Lille Metropole Communaute Urbaine anunciaba con júbilo la instalación de la empresa Kipsta del grupo Oxylane -antes Décatlhon- en el sitio de la Unión ${ }^{33}$, pese a que algunas de sus demandas contradicen al proyecto de ecobarrio como más área para sus instalaciones en detrimento del área del parque público o la creación de un estacionamiento de 700 cajones que no estaban previstas en el plan director. Diversos técnicos concuerdan en que Kipsta no tiene lugar en el ecobarrio, sin embargo el arbitraje

32 Según la CERTU (2006, p.9), la concertación es la implicación basada en el trabajo común de las autoridades, técnicos y habitantes del territorio sobre un proyecto público, de acuerdo a un proceso colectivo previo a la decisión, permitiendo establecer lazos más cercanos entre los actores en torno al proyecto y su apropiación y aceptación por parte de todos.

Lille Metropole Communaute Urbaine, 2009. político se enfrenta a los retos políticos y los del ecobarrio, inclinándose a favor de Kipsta debido a que representa un elemento fuerte para el desarrollo de un polo de excelencia ${ }^{34}$.

Por otro lado, ante la premura para la ampliación de la calle de la Unión, que dará servicio al CETI, la SEM realizó en diciembre de 2010 una sesión informativa para anunciar el inicio de los trabajos. Las reacciones por parte de los habitantes fueron inmediatas, "iqué pasará con nuestras casas?, ¿cómo solucionarán el problema de los estacionamientos?", fueron algunas de las preguntas, y las respuestas de los técnicos se enfocaron principalmente a tranquilizar a los habitantes diciéndoles que sus casas no serían afectadas y que el reordenamiento de la calle de la Unión permitiría la convivencia de diferentes formas de desplazamiento (automóviles, bicicletas, peatones) como lo indica la movilidad sustentable. La tensión entre técnicos y autoridades con los habitantes originada por la falta de un proceso de concertación más efectivo en la elaboración del proyecto saltaba a la vista. Situaciones como ésta han generado un sentimiento de decepción por parte de los habitantes

34 Leromain, 2010, p. 83. 
e integrantes de asociaciones, quienes en repetidas ocasiones han expresado que se sienten fuera del proyecto y encuentran difícil apropiarse de él, "a medida del avance de este gran proyecto nos sentimos fuera de él (...). Nos gustaría ser actores de lo que emergerá de la tierra"35. Lo anterior nos indica que en las instancias de mayor jerarquía (en este caso el comité de pilotaje) para la participación, el proceso de concertación tiende a diluirse.

b) Multiplicación de espacios de diálogo y subutilización de los existentes. Después del evento de Las Asambleas de la Unión en $2009^{36}$, surgieron otras propuestas de espacios de diálogo, como el proyecto club de socios, los talleres de coproducción y los grupos de foro de intercambio. Estos nuevos espacios intentan agilizar y mejorar la comunicación entre los diferentes actores y facilitar la concertación entre ellos, sin embargo no hay estrategias claras para su puesta en marcha. El hecho de multiplicar los espacios de diálogo parece ser la respuesta inmediata a las demandas de mayor participación, empero los espacios ya existentes se han subutilizado y sus contenidos suelen ser ligeros y

\section{Deram, 2010.}

36 Este fue el primer año de Las Asambleas de la Unión, y el correspondiente al 2010 se pospuso para junio de 2011. algunas veces sin ningún fruto. Por ejemplo, uno de los temas abordados en los grupos de trabajo fue la elección del proyecto que será La Casa de la Unión $^{37}$ y para ello dichos grupos habían clasificado del uno al cinco los proyectos propuestos (el número uno representaba el mejor proyecto), no obstante el comité de pilotaje escogió el número cinco, aparentemente por razones financieras y durante un largo periodo el proyecto se suspendió.

A decir de los miembros del Colectivo de la Unión (CU), "La SEM produce espacios que no comprometen a las autoridades. Por ejemplo, en Las Asambleas de la Unión nos reunieron en una sala y nos dijeron que iban a impulsar más la participación, sin embargo eso lo hemos escuchado desde el 2007, pero ¿cuándo comenzaremos? No basta con aumentar los espacios, es necesario que haya verdaderos contenidos" (diciembre de 2010).

Así, ante la falta de discusión de temas que puedan modificar directamente el proyecto, los miembros del CU han desarrollado sus propios espacios para discutir algunas de sus demandas y han elaborado un cuaderno de propuestas ${ }^{38}$ que han presentado

37 La Casa de la Unión servirá como espacio para difundir e informar sobre el proyecto y realizar eventos relacionados a él.

38 Este cuaderno contiene ocho proposiciones específicas: inscribir a la Unión en un plan de conjunto, desclavar a la Unión y valorizar el canal, reavivar el borde del canal, integrar los proyectos, imaginar un laberinto-huerta, programar equipamientos para 9000 usuarios, promover inmuebles polivalentes y adoptar la Vivienda Ecológica Compartida -HEP, por sus siglas en francés-(Université Populaire et Citoyenne, 2010). 
ante las autoridades y público en general. Esta situación acrecienta la multiplicación de los espacios de diálogo, desperdiciando los espacios existentes, como los grupos de trabajo, los consejos y comités de barrio, etc., y nos indica la necesidad de la definición de compromisos por parte de todos los actores para hacer funcionar dichos espacios.

c) Los medios de financiamiento. Las expectativas para desarrollar el ecobarrio de la Unión son mayores que las del resto de los proyectos (particularmente en los aspectos medioambientales y la gestión participativa), sin embargo no hay un presupuesto suplementario para la participación ${ }^{39}$. Este hecho representa un desafío y genera conflictos entre las diferentes partes, ya que el proceso participativo demanda mayores medios de financiamiento para su puesta en marcha (porque se deben desarrollar nuevas competencias, nuevos puestos de trabajo, nuevos espacios, etc.). Por ejemplo, el CU demandó financiamiento para tener espacios de trabajo autónomos y para el desarrollo de sus propuestas, y en 2005 recibió como respuesta a estas demandas la disposición de un local $^{40}$, pero no aceptaron porque no tenía los medios para mantenerlo. Así, el trabajo subsecuente dependió de la benevolencia de los miembros del

39 Con la carta ecobarrio se están redefiniendo los criterios de financiamiento para los ecobarrios, ya que el nivel de acción en ellos suele ser mayor que en el resto de los proyectos.

40 El alcalde de Tourcoing (en esa época Jean-Pierre Baldwick) dio esta respuesta inmediata al CU. colectivo quienes tomaron la iniciativa de visitar el ecobarrio de Fribourg, Alemania para aprender de esta experiencia. Uno de los resultados principales de dicha visita fue la elaboración de " $\mathrm{La}$ carta cambiar de era. Por el derecho a cambiar de era”, que presentaron ante las autoridades, y así cuatro años más tarde, en 2009, obtuvieron un convenio de tres años con la SEM. Dicho convenio les aporta 40,000 euros anuales y es considerada como "una batalla ganada" para los del colectivo, pero no es suficiente y deben movilizarse para obtener más recursos en las diferentes instancias, situación que suele ser desgastante y desalentadora para algunos.

De acuerdo a nuestras entrevistas, a pesar de los 5 millones de euros (de los 118 para el desarrollo del proyecto) destinados para la participación y la concertación, éste resulta insuficiente para administrar todos los espacios de diálogo y ayudar a las asociaciones. Ante esta situación, la SEM ha solicitado activar el Fondo de Participación de los Habitantes (FPH) para el 2011, gestión que actualmente se encuentra en proceso.

Podemos observar que a pesar de la existencia de fondos para las acciones participativas, éstos resultan insuficientes y los procesos para 
obtenerlos a veces son difíciles y no son inmediatos, situación que alarga los procesos para elaborar el proyecto.

d) Diferencias lingüísticas entre técnicos y ciudadanos. Si bien es cierto que "para actuar en el espacio es necesario apoyarse sobre una concepción reconocida y legítima del oficio" ${ }^{41}$, también es cierto que "la participación puede constituir una presión a la transparencia de las opciones que alberga la caja negra del proyecto. Ésta permite verdaderos debates técnicos y científicos por medio de los cuales los ciudadanos interrogan los saberes y sus formas de movilización. La experiencia de los usuarios, en particular, puede favorecer una mejor utilización del proyecto, y por lo tanto una gestión más eficaz". ${ }^{42}$

En el caso de la Unión, aunque el desarrollador está consciente de la importancia del reconocimiento del saber ciudadano, en la práctica resulta difícil deshacer la supremacía de los saberes técnicos sobre los profanos y ponerlos en convivencia y mejor aun en armonía, ya que los primeros continúan encerrándose en su conocimiento experto (a veces megalómano). El único ejemplo donde ha existido disposición clara y abierta para concebir el proyecto directamente con los habitantes (aunque recordemos que éste al final quedó fuera de él) ha sido el caso de las casas de las calles Stéphenson

41 Biau y Tapie, 2009, p. 177.

42 Baqué, 2009, p. 26.

216 revista invi № 76 / Noviembre 2012 / Volumen № 27: 203-221 y Tossée pero el resto de los técnicos del proyecto realizan, en general, las propuestas aisladamente. Esta situación ha llevado al CU a contratar a su propio arquitecto para poder expresar sus propuestas y romper las barreras lingüísticas entre ambos actores. Aunque lo anterior duplica el trabajo y demanda mayor presupuesto, el colectivo logró ganar un espacio para su propuesta del laberinto-huerta en el cuaderno de especificaciones de los paisajistas. En realidad esta última ha sido la única propuesta que hasta el año 2010 fue tomada en cuenta en el proyecto. Lo anterior nos muestra la dificultad para romper las barreras entre los habitantes y los técnicos, y los prejuicios y sentimiento de supremacía por parte de los técnicos para reconocer y valorar el conocimiento profano.

e) Marco reglamentario limitante. El proyecto de la Unión se circunscribe al marco legal general para la participación en Francia (Ley sobre la administración territorial (1992), Ley Solidaridad y renovación urbana (2000), Ley Voynet (1999) y Ley Vaillant (2002) y a las iniciativas locales como la Agenda 21 de Lille (2006) y la Carta Ecobarrios (2010). Este marco legal ha servido para obligar a las autoridades y a sus técnicos a desarrollar proyectos abriendo la puerta a la participación, no obstante como lo señala Drobenko ${ }^{43}$, un limite muy importante en el proceso participativo es

43 Drobenko, 2010. 
que la consulta pública no obliga a la autoridad que tomará las decisiones a seguir las conclusiones que elaboran los comisarios encargados de dicha consulta. Por su parte la Carta ecobarrios tampoco obliga a las autoridades a tomar en cuenta de ninguna manera los resultados de los grupos de trabajo.

Los ciudadanos solicitan en esta materia que las autoridades reconozcan que los ciudadanos son capaces de defender los intereses generales y tener una visión amplia del proyecto, ya que esta postura ayudaría a romper con las barreras reglamentarias en torno a la participación.

f) Un proyecto intercomunal difícil de gestionar. En el ecobarrio de la Unión, la cooperación intercomunal es percibida como una condición para el éxito del proyecto. "La originalidad del pilotaje político de la Unión radica en que en el seno del proyecto las autoridades de las comunas de Roubaix, Tourcoing y Wattrelos asumen a la vez el rol de alcalde y de vicepresidente intercomunal"44. Cada uno tiene una competencia específica dentro del proyecto: la economía corresponde al alcalde de Tourcoing (Michel François Delannoy), el ordenamiento del territorio al de Roubaix (René Vandierendonck) y las finanzas al de Wattrelos (Dominique Baert). Este doble rol de los alcaldes los pone en una situación de conflicto, ya que por un lado

44 Leromain, 2010, p.90. deben procurar el mejor desarrollo para su comuna y al mismo tiempo encontrar un equilibrio para el desarrollo del proyecto intercomunal. Los intereses particulares que cada alcalde defiende para su comuna y la falta de una autoridad intercomunal exterior al proyecto pone en duda la verdadera voluntad política intercomunal.

Aunado a lo anterior, la LMCU, que es responsable del proyecto de la ZAC, no se siente con la legitimidad para responder a las demandas de los ciudadanos debido a que no es elegida en sufragio universal. Esta falta de legitimidad de la Comunidad Urbana desemboca en los vaivenes entre los alcaldes y la LMCU de los que son objeto las asociaciones. El Colectivo de la Unión, por ejemplo, difícilmente encuentra una respuesta a sus peticiones ante la LMCU pues ésta delega la responsabilidad a los diferentes alcaldes (miembros de la intercomuna) y ellos a su vez la delegan a la LMCU, convirtiendo el proceso en un círculo vicioso.

La aparente falta de voluntad política intercomunal y la falta de legitimidad sobre el proyecto desemboca en un doble juego para los ciudadanos, ya que por un lado provoca vaivenes que los llevan de una autoridad a otra y por el otro les da un margen de maniobra que les permite negociar sus demandas. 


\section{Conclusiones}

El proyecto de ecobarrio de la Unión representa muy bien las intenciones políticas de aplicar los principios de desarrollo sustentable en un escenario decisional compartido con los ciudadanos y al mismo tiempo el interés creciente de estos últimos de formar parte en los procesos participativos que buscan construir la ciudad de manera conjunta y diferente. Estos procesos implican pasar del discurso a la práctica donde es fácil caer en contradicciones ante la dificultad de romper con los paradigmas establecidos en los modelos decisionales burocráticos establecidos desde hace tiempo, donde el "Estado tiene un rol de portador del interés general, según un modelo de ejecución mecanicista e instrumental" 45 .

La percepción sobre la gobernabilidad del proyecto es distinta por parte de los diferentes actores, ya que por un lado las autoridades argumentan que se trata de un proyecto abierto y participativo y por el otro los ciudadanos sienten una fuerte dificultad para influir en el proyecto. Sin embargo, ambas partes reconocen la necesidad de establecer relaciones de diálogo conjuntas para la toma de decisiones respecto al desarrollo del ecobarrio.

Hasta ahora, los dispositivos y espacios de diálogo para alentar la participación y la concertación

45 Gauthier y Lépage, 2005, p.108.

218 revista invi № 76 / Noviembre 2012 / Volumen N ${ }^{0} 27:$ 203-221 sobrepasan los procesos tradicionales de elaboración de los proyectos y se han convertido en una necesidad para construir la ciudad, una demanda en aumento por parte de la población y un objetivo político. Así, la multiplicación de los espacios para la participación resulta un argumento político en favor de la gestión participativa, sin embargo la falta de una escucha empática y la subutilización de dichos espacios debilitan su objetivo, ubicando al proceso participativo en una etapa de información-consulta con un lento ascenso hacia la concertación. Esta experiencia nos muestra que a pesar de la disponibilidad para elaborar el proyecto conjuntamente por parte de las autoridades y habitantes, los intereses políticos tienden a ser más fuertes que los intereses comunes, no obstante existe una lucha constante por parte de los habitantes para hacer prevalecer el interés común. No debemos olvidar que, como lo indica Callon ${ }^{46}$, tanto el experto como el voluntarismo político en cuanto adquieren un discurso de autoridad, fracasan en responder a las interrogantes de los ciudadanos. Toda tentativa para ignorar la fecundidad de las discusiones y reducir los debates a simples formalidades o meterlos en una camisa de fuerza, tarde o temprano se vuelve contra quienes pretenden conocerlo todo. Por lo anterior resulta importante reconocer el conocimiento ciudadano pues ello permitiría romper las barreras entre la

46 Callon, 2001. 
participación ciudadana reglamentaria y su verdadera implementación. En este sentido, uno de los aportes de esta experiencia es la iniciativa de los habitantes de la Unión para la creación de espacios de diálogo independientes de los oficiales que coadyuvan a hacer más efectivas las discusiones con las autoridades locales en los espacios oficiales, ya que las ideas y propuestas por parte de los habitantes suelen ser más claras y objetivas. Esto pone de relieve el cambio de mentalidad de los habitantes, reflejado, entre otras características, en su constancia y empeño por realizar cambios y apropiarse del proyecto a través de iniciativas como la citada anteriormente.

Por otro lado, el ecobarrio de la Unión pone en evidencia la inexistencia de fórmulas preestablecidas para desarrollar un proyecto con gestión participativa y al mismo tiempo nos aporta algunos elementos fundamentales para la realización del ejercicio participativo, como la iniciativa por parte de las autoridades locales para la creación de los espacios de diálogo, la necesidad de formar gestores para el proyecto urbano con participación social, las subvenciones para desarrollar el trabajo en las asociaciones, el trabajo voluntario de los habitantes, un marco legal claro y contar con un presupuesto participativo dentro del proyecto.

Finalmente, ante la falta de legitimidad de la LMCU, la relación efectiva entre la Comunidad Urbana con los ciudadanos parece dudosa. Mientras tanto, las controversias, entre los diferentes actores, en los espacios de diálogo existentes para el desarrollo e implementación del ecobarrio de la Unión, están permitiendo abrir un camino más amplio para avanzar en la construcción de una gestión participativa.

\section{Bibliografía}

ASCHER, François. Los nuevos principios del urbanismo. $2^{a}$ ed. Madrid, España, Alianza. 2007. 93 p. ISBN 978-84-206-4198-0.

BACQUE, Marie-Hélène. Gouvernance et urbanisme de participation. En: BIAU, Véronique y TAPIE, Guy, dir. La fabrication de la ville. Métiers et organisations. Marsella, Francia, Parenthèses, 2009. p. 17-29. ISBN 978-2-86364-651-9. Collection eupalinos.

BIAU, Véronique y TAPIE, Guy, dir. La fabrication de la ville. Métiers et organisations. Marsella, Francia, Parenthèses. 2009. 220 p. ISBN 978-2-86364651-9. Collection eupalinos.

CALLON, Michel; LASCOUMES, Pierre y BARTHE, Yannick. Agir dans un monde incertain. Essai sur la démocratie technique. París, Francia, Du Seuil. 2001. 358 p. ISBN 978-2-02-040432-7.

CERTU (Centre D'études Sur les Réseaux, les Transport, l'urbanisme et les Constructions Publiques). La concertation, Cour du développement durable. Francia, CERTU. 2006. 35 p. ISBN 2-11-095328-4.

revista invi № 76 / Noviembre 2012 / Volumen № 27: 203-221 219 
CHARLOT-VALDIEU, Catherine y OUTREQUIN, Philippe. Écoquartier mode d'emploi. París, Francia, Eyrolles. 2009. 243 p. ISBN 978-2-212-12601-3.

DA CUNHA, Antonio. Développement durable, transformations urbaines et projet: enjeux et défis. En: DA CUNHA, Antonio; KNOEPFEL, Peter; LERESCHE, Jean-Philipe y NAHRATH, Stéphane. Enjeux du développement urbain durable. Transformations urbaines, gestion des ressources et gouvernance. Laussane, Suiza, Les presses polytechniques et universitaires romandes. 2005. p. 9-11. ISBN 2-88074-652-3.

DERAM, Bruno. Zone de l'Union: le collectif d'associations s'estime "mis de côte". [En línea]. La Voix du Nord. 24 de abril de 2010. [Fecha de consulta: 27 de septiembre de 2011]. Disponible en: http://www.lavoixdunord.fr/Locales/Tourcoing/actualite/Secteur_Tourcoing/2010/04/24/ article_zone-de-l-union-le-collectif-d-associati. shtml?xtor=RSS-2.

DROBENKO, Bernard. Tour d'horizon du droit qui fonde la concertation. [En línea]. CDE - Colloques. 26 de febrero de 2009. [Fecha de consulta: 08 de octubre de 2011]. Disponible en: http:// www.concertation-environnement.fr/index. php?option=com_content\&task=view\&id=85\&It emid $=44$.

GAUTHIER, Mario y LEPAGE, Laurent. La mise en ouvre de la ville viable. En: MATHIEU, Nicole y GUERMOND, Yves. La ville durable, du politique au scientifique. París, Francia, Editions Quae. 2005. p. 101-117. ISBN 2-7380-1202-7.

220 revista invi № 76 / Noviembre 2012 / Volumen N ${ }^{0}$ 27: 203-221
GOBIERNO de Francia. L'appel à projets 2009 du Ministère. [En línea]. Ministère du Développement durable. 30 noviembre 201la. Disponible en: http:// www.developpement-durable.gouv.fr/L-appel-aprojets-du-Ministere.html.

GOBIERNO de Francia. Le Grenelle Environnement. [En línea]. 2011b. [Fecha de consulta: 6 enero 2012]. Disponible en: http://www.legrenelle-environnement.fr.

HUGH, Barton. Sustainable communities. The potential for eco-neighbourhooods. Londres, Inglaterra, Earthscan. 2000. 305 p. ISBN 1853835137.

IRACHETA, Alfonso. Gobernanza y desarrollo local, nuevo camino para la sustentabilidad. [En línea]. EDRA (39², 2008, Boca del Río Veracruz). Linking Differences/Defining Actions. Proceedings of the 39th Annual Conference of the Environmental Design Research Association. p. 12-20. ISBN 0-939922-32-0. Disponible en: http://www.edra. org/sites/default/files/publications/EDRA39-Cenecorta_1.pdf.

LA UNIÓN. Site du Projet de l’Union. [En línea]. L'UNION - Renouvellement urbain, ville durable en métropole lilloise. 2011. [Fecha de consulta: 08 de noviembre de 2011]. Disponible en: http://www. lunion.org.

LEFÈVRE, Pierre y SABARD, Michel. Les écoquartiers. Rennes, Francia, Apogée. 2009. 261 p. ISBN 978-2-84398-325-2.

LEROMAIN, Clémence. L'Union, Pôle d'excellence et écoquartier. Analyse d'un projet intercommunal.

ARTíCULO: En busca de la gestión participativa: el caso del proyecto del Ecobarrio de la Unión, Lille, Francia / María de Lourdes Flores Lucero 
Mémoire de Maîtrise. Memoria de Máster 1 Aménagement Urbanisme et Développement des territoires. Spécialité Construction Aménagement Durable. Institut d'Aménagement et Urbanisme de Lille. 2010. 128 p.

LILLE Metropole Communaute Urbaine (LMCU). Le portail de Lille Métropole. [En línea]. Lille Métropole. 2009. [Fecha de consulta: 6 de enero de 2011]. Disponible en: http://www.lillemetropole. fr.

MORIN, Edgar. La Voie. Pour l'avenir de l'humanité. Francia, Fayard. 2011. 312 p. ISBN 978-2-213-65560-4.

SIMON, Jean-Marie. Concertés parce que concernés. [En línea]. MINISTĖRE de l'écologie et du développement durable et Secrétariat d'État au développement durable. Les outils et démarches en vue de la réalisation d'agendas 21 locaux. Parole aux acteurs. 2003 p. 39-40. Dossier documentaire No. 3. [Fecha de consulta: 05 de marzo de 2012]. Disponible en: http://www.cidce.org/3dmac/documentation/paroles\%20acteurs.pdf.

SOCIÉTÉ d’Economie Mixte (SEM). Stéphenson, la refonte d'un quartier. France, Turcoing, L'Union Lille Metropole. 2010. 26 p. Les livrets de l'Union $\mathrm{N}^{\circ} 2$.
SOUAMI, Taoufik. Écoquartiers, secrets de fabrication. Analyse critique d'exemples européens. París, Francia, Les Carnets de l'info. 2009. 207 p. ISBN 978-2-9166-2844-8

TAMAYO, Sergio. La práctica de la ciudadanía en la redefinición de la modernidad y las utopías urbanas. En: TAMAYO, Sergio coord. Sistemas urbanos. Actores sociales y ciudadanías. México D.F., UAM-Azcapotzalco. 1998. p. 131-163. ISBN 970-654-217-5.

UNIVERSITÉ Populaire et Citoyenne (UPC). 10 propositions pour l'Union. [En línea]. UPC à Roubaix. Octubre de 2010. [Fecha de consulta: 5 de marzo de 2012]. Disponible en: http://upc-roubaix.org/ IMG/pdf/collectif_de_1_union_propositions_10_ decembre_2010.pdf.

UNIVERSITÉ Populaire et Citoyenne (UPC). De l'Alma à l'Union, vers une éco-quartier exemplaire? [En línea]. Les cahiers de l'UPC à Roubaix. (30), febrero 2011. ISSN 1778-039X. [Fecha de consulta: 25 de febrero de 2012]. Disponible en: http://upc-roubaix.org/IMG/pdf/cahier30bd3.pdf.

ZICCARDI, Alicia. La tarea de gobernar: las ciudades y la gobernabilidad. En: ZICCARDI, Alicia coord. La tarea de gobernar: gobiernos locales y demandas ciudadanas. México D.F. Porrúa. 1996. p. 1337. ISBN 968-842-545-1. 